\title{
Multipotent adult progenitor cell transplantation increases vascularity and improves left ventricular function after myocardial infarction
}

\author{
Beatriz Pelacho ${ }^{1,3}$, Yasuhiro Nakamura ${ }^{2}$, Jianyi Zhang ${ }^{2}$, Jeff Ross ${ }^{1}$, Yves Heremans ${ }^{1}$, \\ Molly Nelson-Holte ${ }^{1}$, Brad Lemke ${ }^{1}$, Julianna Hagenbrock ${ }^{1}$, Yuehua Jiang ${ }^{1}$, Felipe Prosper ${ }^{3}$, \\ Aernout Luttun ${ }^{1}$ and Catherine M. Verfaillie ${ }^{1 * \#}$ \\ ${ }^{1}$ Stem Cell Institute, University of Minnesota Medical School, Minneapolis, MN 55455, USA \\ ${ }^{2}$ Division of Cardiology, Department of Medicine, University of Minnesota Medical School, Minneapolis, MN 55455, USA \\ ${ }^{3}$ Haematology and Cell Therapy, Clinica Universitaria, Av Pio XII 36, Pamplona 31008, Spain
}

\begin{abstract}
Progressive contractile dysfunction of viable myocardium that surrounds a large infarct leads to heart failure following acute myocardial infarction (AMI). Experimental evidence indicates that cellular transplantation may improve the left ventricular (LV) contractile performance, even though the underlying mechanisms remain undefined. Here, we compared the effect of transplantation of murine multipotent adult progenitor cells (MAPCs), a population of adult bone marrow-derived cells that differentiate into cells of mesodermal, endodermal and ectodermal origin, with murine bone marrow cells (BMCs) or fibroblasts on post-infarct cardiac function by peri-infarct injection after coronary artery ligation in mice. We demonstrate that, in contrast to the other cell populations, transplantation of MAPCs significantly improved LV contractile function for at least 8 weeks posttransplantation and, although BMCs reduced infarct size, the decrease in scar size was substantially greater in MAPC-treated hearts. As neither MAPCs nor BMCs were present beyond 1 week, the beneficial effect was not due to differentiation and direct contribution of MAPCs to the vascular or cardiomyocyte compartment. Significantly more inflammatory cells were present in MAPC- than BMC-treated hearts at 1 week, which was accompanied by increased vascularity 8 weeks posttransplantation. We hypothesize that MAPCs indirectly contributed to these effects, by secreting inflammatory [monocyte chemoattractant protein-1 (MCP)-1], and vascular growth factors [vascular endothelial growth factor (VEGF), platelet-derived growth factor (PDGF)-BB, and transforming growth factor (TGF) $\beta_{1}$ ), and others, resulting in increased angiogenensis and cardioprotection. Copyright $\odot 2007$ John Wiley \& Sons, Ltd.
\end{abstract}

Received 11 December 2006; Revised 15 January 2007; Accepted 30 January 2007

Keywords adult stem cells; bone marrow; acute myocardial infarction; angiogenesis; cellular therapy

\section{Introduction}

Cardiovascular disease is now the leading cause of death worldwide (Chockalingam et al., 2000). While there

\footnotetext{
*Correspondence to: Catherine M. Verfaillie, Stamcel Instituut Leuven, Katholieke Universiteit Leuven, UZ Gasthuisberg, Herestraat 49, 3000 Leuven, Belgium.

E-mail: catherine.verfaillie@med.kuleuven.be

\# Conflict of interest: C. M. Verfaillie is a consultant to Athersys Inc., Cleveland, $\mathrm{OH}$, USA.
}

has been a reduction in mortality of acute myocardial infarction (AMI), a significant proportion of patients proceed to heart failure, which affects 5 million people in the USA and 22 million people worldwide (Chockalingam et al., 2000). Heart failure occurs when injury to the myocardium results in cardiomyocyte death, followed by replacement of injured myocardium by fibrous tissue, scar expansion, inability of the viable myocardium to compensate for the failure of the injured region, and activation of compensatory mechanisms. Eventually, these 
factors lead to increased myocardial wall stress, with changes in cardiomyocyte size, metabolism, and gene expression, as well as ventricular dilatation and ultimately reduced systolic function. Collectively this process is known as myocardial remodelling and is the phenotypic hallmark of heart failure (Francis and Tang, 2003). As the treatment options are extremely limited, the ultimate goal remains to prevent these complications by repairing the myocardium when injury occurs. Stem or progenitor cell transplantation is a twenty-first-century approach that offers the first real potential for significant cardiac repair and recovery.

An ideal cell population for repairing the ischaemic heart should be capable of: (a) myogenesis, i.e. specifically generate cardiomyocytes that integrate with the native myocytes, form gap junctions, and achieve electromechanical coupling; (b) neovascularization, i.e. restore blood supply to the viable myocardium at risk and provide oxygen and nutrients to the grafted cardiomyocytes; and (c) positively affect regional and global ventricular remodelling, geometry and wall stress. Several cell populations have been used in animal models of AMI, some of which are also being used clinically. For instance, skeletal myoblasts are myogenic but cannot differentiate into endothelial cells (ECs). However, skeletal myoblasts have failed to specifically differentiate into cardiomyocytes that are fully integrated and electrically connected to adjacent cardiomyocytes, rendering the ventricle susceptible to electrical instability and arrhythmias (Menasche, 2005). Mesenchymal stem cells (MSCs) have myogenic ability, although there is no good proof that they can differentiate into functional cardiomyocytes (Tomita et al., 1999; Davani et al., 2003; Mangi et al., 2003). On the other hand, unfractionated bone marrow cells (BMCs) or bone marrow (BM) enriched for $\mathrm{CD}^{+} 4^{+}$or $\mathrm{cKit}^{+}$cells have been proposed as potential cell populations for cardiac repair because of their angiogenic potential (Jackson et al., 2001; Kamihata et al., 2001). Also, the contribution of these cells to myogenesis has been shown (Orlic et al., 2001; Kajstura et al., 2005); however, some controversy exists on this (Jackson et al., 2001; Balsam et al., 2004; Murry et al., 2004).

Finally, endothelial progenitor cells (EPCs) (Kocher et al., 2001) and meso-angioblasts (Galli et al., 2005) have angiogenic but not myogenic capabilities.

We isolated a subpopulation of BMCs known as multipotent adult progenitor cells (MAPCs), which differentiate into most, if not all, somatic cell types. MAPCs differentiate into vascular cell types, including ECs and smooth muscle cells (SMCs) in vitro and in vivo (Jiang et al., 2002). Although MAPCs contribute to the heart when injected into the blastocyst (Jiang et al., 2002), we have not investigated whether they do so in a pathological situation such as AMI. Therefore, in the current study, we compared the effect of mouse MAPCs with mouse BMCs and fibroblasts (MEFs), grafted shortly after left anterior descending coronary artery (LAD) ligation, on the histology and function of the murine heart for up to 8 weeks following transplantation.

\section{Methods}

An extended methods section is available on-line.

\subsection{Mouse strains}

C57BL/6 and C57BL/6-(ROSA)26 mice (Jackson Laboratory, Bar Harbor, MA) were housed under specific pathogen-free conditions. All protocols involving mice were approved by the Institutional Animal Care and Use Committee (IACUC).

\subsection{MEF and $B M C$ isolation}

ROSA26-MEFs were isolated from embryonic day (E)14 pups, as described (Hogan, 1994). ROSA26-BMCs were harvested from adult mouse femurs and red blood cells depleted by ammonium chloride treatment.

\subsection{MAPC isolation and characterization}

ROSA26-MAPCs were isolated from adult mouse BM, subcloned at 10 cells/well and expanded as described (Jiang et al., 2002). Karyotype was detemined as described (Jiang et al., 2002), revealing that the cells were karyotypically stable throughout the study. Expression of pluripotency markers Oct4 and Rex1 was analysed by quantitative (Q) RT-PCR (for primers, see Supplementary Table 1), showing that the cells expressed low levels (>1000-fold less than mouse embryonic stem cells) of both markers. To demonstrate tri-lineage differentiation potential, the cells were differentiated to endothelial (respresenting mesoderm; see below), hepatocyte-like (Jiang et al., 2002) (representing endoderm), and neuronlike cells (Jiang et al., 2002) (representing ectoderm) and evaluated by Q-RT-PCR.

\subsubsection{For EC differentiation}

MAPCs $\left(45 \times 10^{4}\right.$ cells $\left./ \mathrm{cm}^{2}\right)$ were plated on fibronectin (FN; Sigma)-coated wells in serum-free DMEM/MCDB (Sigma) with $10 \mathrm{ng} / \mathrm{ml} \mathrm{VEGF}_{165}$ (R\&D Systems) (Reyes et al., 2002). On day 14, expression of platelet endothelial cell adhesion molecule (PECAM), von Willebrand Factor (vWF), VE-cadherin (VEC), Flt-1 and Tie-2 was analysed by Q-RT-PCR (for primers, see Supplementary Table 1) and immunostaining for vWF and VEC.

\subsubsection{For SMC differentiation}

MAPCs $\left(2 \times 10^{3}\right.$ cells $\left./ \mathrm{cm}^{2}\right)$ were plated on FN-coated wells in serum-free DMEM/MCDB with $2.5 \mathrm{ng} / \mathrm{ml}$ TGF $\beta 1$ and $5 \mathrm{ng} / \mathrm{ml}$ PDGF-BB (R\&D Systems). On day 6, expression of smooth muscle $22 \alpha$ (SM22 $\alpha$ ), calponin and $\alpha$-smooth muscle actin ( $\alpha$-SMA), was analysed by 
Q-RT-PCR (for primers, see Supplementary Table 1) and immunostained for $\alpha$-SMA and SM22 $\alpha$.

\subsubsection{For cardiomyocyte differentiation}

MAPCs $\left(30 \times 10^{3}\right.$ cells $\left./ \mathrm{cm}^{2}\right)$ were plated on FN/gelatincoated wells, in serum-free DMEM/MCDB with $10 \mathrm{ng} / \mathrm{ml}$ activin and DKK-1 (R\&D Systems) and $3 \mu \mathrm{M}$ 5Aza2'deoxycitidine (Sigma). On day 6, Q-RT-PCR for Nkx2.5, GATA6, MEF2C and Tbx5 (for primers, see Supplementary Table 1) was performed.

\subsection{ELISAs}

After $60 \mathrm{~h}$ of incubation, the supernatants of MAPCs, MEFs and BMCs and control media were collected and frozen. Murine MCP-1, VEGF, PDGF-BB and TGF $\beta_{1}$ were measured by ELISA (R\&D Systems, Minneapolis, MN) according to the manufacturer's instructions.

To assess the degree of cardiomyocyte apoptosis, we used an ELISA kit (Roche Applied Science, Spain) in which oligonucleosomal fragments are quantified.

\subsection{EC and SMC proliferation assay}

The murine EC line (MS-I) (ATCC, Manassas, VA) and SMC line (Cell Applications, San Diego, CA) were cultured in the presence of MAPC-conditioned media (CM) or non-conditioned medium (NCM) for $60 \mathrm{~h}$. The number of viable cells was quantified using a Nucleocounter (Chemometec, Denmark).

\subsection{Animal surgery, intracardiac cell injection and cardiac echocardiography}

A total of 50 13-16 week-old mice were pre-/posttreated with anti-asialo GM1 antibody (Wako, Chemicals, Japan). An infarct was induced by left descending coronary artery (LAD) ligation as previously described (Nakamura et al., 2002) and $0.5 \times 10^{6}$ MAPCs, BMCs or MEFs resuspended in $60 \mu \mathrm{L}$ DMEM, or DMEM alone, were injected in three distinct peri-infarct sites in the myocardium. Mortality due to surgery was $<25 \%$. Mice underwent cardiac ultrasound on days $-1,+1$, 7, 14, 28 and 56, as previously described (Nakamura et al., 2002). The left ventricular (LV) ejection fraction (EF) and fractional shortening (FS) were calculated as follows: $\mathrm{EF}=\left(\mathrm{LVDd}^{2}-\mathrm{LVDs}^{2}\right) / \mathrm{LVDd}^{2} \times 100$; and $\mathrm{FS}=$ (LVDd - LVDs)/LVDd × 100 .

\subsection{Tissue processing, (immuno)staining and morphometric analysis}

Infarcted mice were anaesthetized, perfusion-fixed, and the excised hearts were stained for LacZ and processed for histology. Antibody sources were: Bandereia simplifolia (BS)-I lectin (Sigma, St Louis, MO); anti-CD45 (BioLegend, San Diego, CA); anti- $\alpha$-SMA-Cy3 (Sigma); and anti-cardiac specific $\alpha$-MHC (Abcam, Cambridge, MA). Staining with haematoxylin and eosin (H\&E) and Sirius red was performed as described (Heymans et al., 1999). Morphometric analysis was performed as described in the Supplementary Methods.

\subsection{Statistical analysis}

All data are expressed as mean \pm SEM and comparisons between two groups were analysed by Student's $t$-test. Comparisons among three or more groups were analysed by ANOVA test. Shapiro-Wilk test was used to verify that the data had a Gaussian distribution, which justified the use of a parametric test. SPSS 12 software was used for statistical analyses and differences were considered statistically significant when $p<0.05$.

\section{Results and Discussion}

The availability of direct comparative cell transplantation studies in AMI models from the literature is limited. Therefore, the current study was designed to compare the effect of MAPCs on the acute infarcted heart with that of two other cell populations. BMCs were used as 'positive' control, as they contain haematopoietic cells, EPCs and mesenchymal stem cells (MSCs), all of which have been shown to affect cardiac function following AMI (Jackson et al., 2001; Kamihata et al., 2001; Kocher et al., 2001). Moreover, BMCs are already being used in ischaemic patients, where they have shown variable effects following intramyocardial or intracoronary transplantation (Murry et al., 2005). The second population, fibroblasts, were used as a presumably negative control. All animals in the study were pre- and post-treated with an anti-NK antibody to avoid NK-mediated immune rejection of MAPCs, which express only low levels of MHC-I (Tolar et al., 2006). Shortly following ligation of the LAD, DMEM vehicle control, $0.5 \times 10^{6}$ MAPCs, BMCs or MEFs, were injected in three locations in the peri-infarct zone. The procedure caused overall $<25 \%$ mortality.

Measurement of the scar area 8 weeks post-MI demonstrated that transplantation of MAPCs or BMCs, but not MEFs, significantly reduced the scar area compared to vehicle control (scar area expressed as a percentage of the left ventricle (LV) area: $50.7 \pm 3.8 \%$ for DMEM, $44.2 \pm 6.7 \%$ for MEFs, $35.7 \pm 2.3 \%$ for BMCs and $21.0 \pm 2.9 \%$ for MAPCs; $P=\mathrm{NS}, p<0.05$, $p<0.05$, respectively, vs. DMEM; Figure $1 \mathrm{~A}-\mathrm{H}$ ). Scar size reduction in MAPC-transplanted hearts was greater than in hearts grafted with BMCs, although this did not reach significance $(p=0.078)$. To assess the effect of cell injection on cardiac function, serial cardiac echography was performed. Comparison of day 1 post-infarct with preinfarct readings revealed that LAD ligation decreased the ejection fraction (EF) from $58.9 \pm 0.3 \%$ to $33.7 \pm 0.2 \%$ 

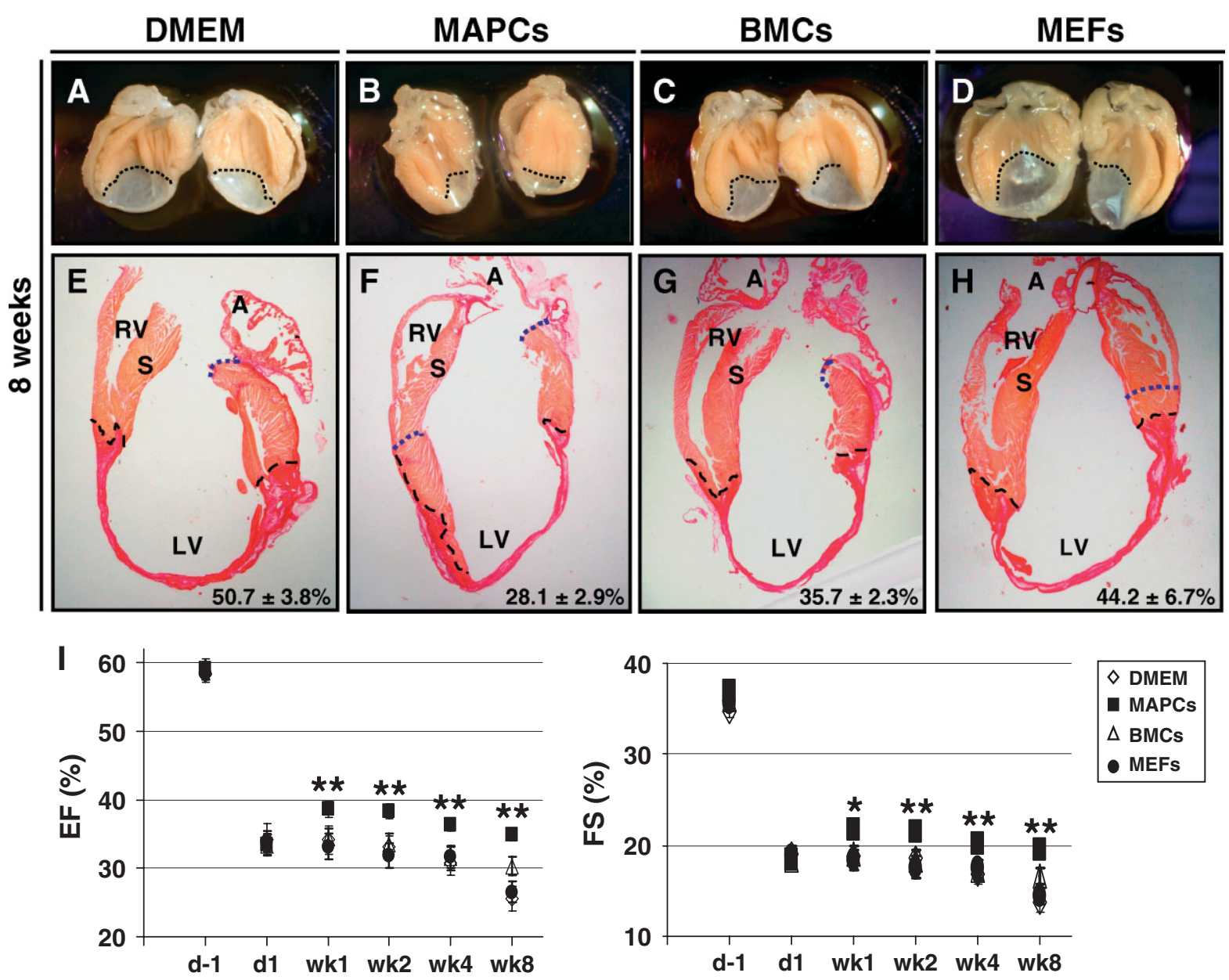

Figure 1. MAPCs significantly reduce infarct size and durably improve EF and FS after AMI. (A-H) Macroscopic evaluation 8 weeks after AMI revealed that scar size (demarcated by black dotted lines) was significantly smaller in MAPC- (B) and BMC-treated hearts (C) vs. DMEM- (A) or MEF-treated (D) hearts. Morphometric analysis on Sirius red-stained sections (E-H) confirmed a significant decrease in infarct size in MAPC (F) and BMC- (G) but not MEF-treated (H) vs. DMEM-treated (E) hearts. Size of the scar (demarcated by red dotted lines) in panels E-H is expressed as mean $\% \pm$ SEM of the total LV area (border demarcated by blue dotted lines). LV, left ventricle cavity; RV, right ventricle cavity; A, atrium; S, septum. (I) Ejection fraction (EF) and fractional shortening (FS) parameters were measured by echocardiography before (d -1) and after infarction (d1, wk1, wk2, wk4 and wk8; where $d=$ day and $w k=$ week). Results, shown as mean \pm SEM for each time point, reveal a significant and sustained increase, as compared to DMEM, in both parameters for MAPC-treated mice but not in the other cell groups. Readings at day 1 post-MI indicated that infarcts were similarly induced in all groups. ${ }^{*} p<0.05$ vs. DMEM. ${ }^{* *} p<0.01$ vs. DMEM

and the fractional shortening (FS) from $35.8 \pm 0.4 \%$ to $18.6 \pm 0.2 \%$, similarly in all treatment groups. No significant variability among groups at day 1 postinfarction was detected ( $p=0.80$ for $\mathrm{FE} ; p=0.81$ for FS; one-way ANOVA), meaning that similar infarcts were provoked in all animals. In the vehicle group, the EF and FS remained stable at 2 weeks, but the EF further decreased to $30.7 \%$ and $26.6 \%$ at weeks 4 and 8 respectively, and the FS decreased to $16.6 \%$ and $13.8 \%$ at those time points, consistent with progressive cardiac failure. At any time point after MEF transplantation, both parameters were not different from the vehicle control group (Figure 1I,J). In BMC-grafted mice, loss in EF and FS between weeks 1 and 8 was less pronounced but the differences with vehicle control or MEF-grafted animals were not statistically significant (Figure 1I,J). In contrast, EF and FS of MAPC-transplanted hearts were significantly higher than the vehicle control group as early as 1 week after transplantation, an effect that persisted for the duration of the study (Figure 1I,J).

To evaluate possible mechanisms underlying the better functional and anatomical preservation of the infarcted heart by MAPC transplantation, histological evaluation was performed. Unlike MEFs, MAPCs and BMCs engrafted the $\mathrm{LV}$, as evidenced by the presence of $\mathrm{LacZ}^{+}$cells in the periphery of the infarct 7 days post-injection (Figure 2A-D). However, the cells disappeared by the second week. Our finding that no BMCs could be detected beyond 1 week after transplantation is at variance with some reports indicating that at least some transplanted cells can be detected up to 6-8 weeks after grafting in the ischaemic heart (Jackson et al., 2001; Mangi et al., 2003; Murry et al., 2004). However, recovery was usually $<1 \%$ in those studies. In addition, as previously reported, detection of $\beta$-galactosidase ${ }^{+}$cells is more difficult than green fluorescent protein (GFP)-labelled 


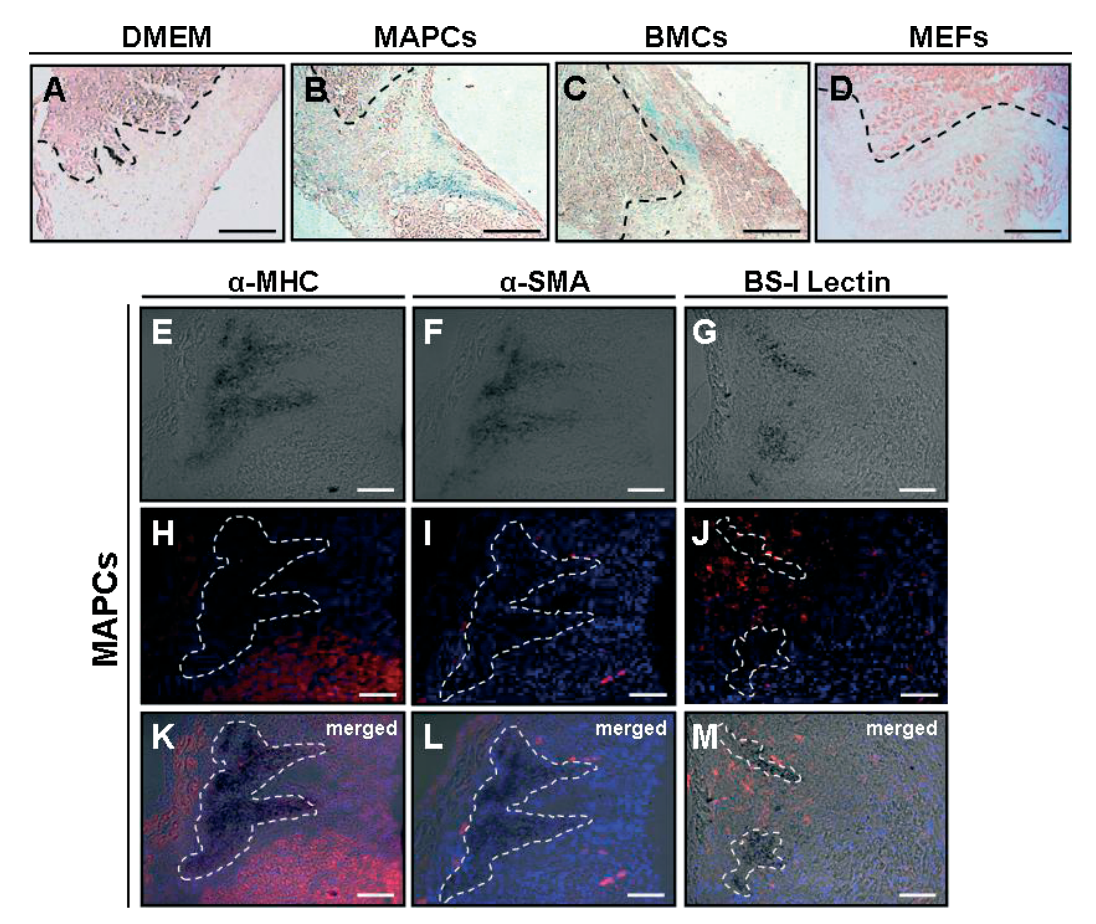

Figure 2. MAPCs and BMCs engraft but do not differentiate into vascular or cardiac muscle cells in the infarcted heart. (A-D) Engraftment. Eosin-counterstained sections of LacZ (blue) stained-hearts, injected with DMEM (A), MAPCs (B), BMCs (C) or MEFs (D) 1 week after infarction, revealing engraftment of LacZ ${ }^{+}$cells in the peri-infarct zone in MAPC and BMC but not MEF or DMEM groups. Black dotted lines in (A-D) delineate the infarct border. Scale bars in (A-D) represent $0.5 \mathrm{~mm}$. (E-M) Differentiation. Staining for cardiac-specific $\alpha$-myosin heavy chain $(\alpha$-MHC) for cardiomyocytes (H), $\alpha$-SMA for SMCs (I) or BS-I lectin for ECs $(\mathrm{J})$ in red on sections, previously stained for LacZ (see dark areas in E-G visualized in phase contrast) from mouse hearts 7 days post-transplantation with MAPCs. The merged pictures (K-M) reveal no co-localization of transplanted LacZ ${ }^{+}$cells with any of the markers. However, there was a close spatial relationship between vessels and grafted cells (I, J, L, M). Dotted lines in (H-M) delineate the area where the grafted cells are located. Nuclear staining (blue), DAPI. Scale bars in (E-M) represent $100 \mu \mathrm{m}$

cells, which may have led to an underestimation of rare surviving cells in our studies (Brazelton and Blau, 2005). Loss of $\beta$-galactosidase expression was not due to its previously documented heterogeneous expression in ROSA26 animals (Kisseberth et al., 1999), since PCR analysis for the $\beta$-Geo gene of sections spanning the entire heart tissue revealed little, if any, gene copies (data not shown). Finally, it is well known that neoantigens, such as neomycin resistance gene, $\beta$-galactosidase or GFP, can serve as immunogens and cause rejection (Andersson et al., 2003). Our preliminary studies in which MAPCs and BMCs from ROSA26 or eGFP transgenic mice were grafted in immune-deficient IL $2 \gamma \mathrm{CR}^{-/-}$Rag $2^{-/-}$animals have shown that both cell populations can be detected for at least 15 days after transplantation, suggesting that, in the current study, some degree of immune rejection may have eliminated $\beta$-galactosidase ${ }^{+}$cells following transplantation. This is consistent with studies by Tolar et al. (2006), that demonstrate that MAPCs are rejected via both an NK- and a T-cell mediated mechanism.

Despite their inherent capacity to differentiate into ECs and SMCs in vitro (Supplementary Figure 1) or their ability to contribute to ECs in tumours in vivo (Reyes et al., 2002), double-staining for LacZ and $\alpha$-SMA or (BS)-I lectin, revealed that in the infarcted heart LacZ ${ }^{+}$MAPC did not co-localize with vascular cell markers (Figure 2L,M). Also, BMCs, known to contain EC and SMC precursors (Shimizu et al., 2001; Urbich and Dimmeler, 2004), did not directly incorporate into blood vessels (not shown). Possibly, their short-term engraftment within the necrotic environment of the infarcted heart may have prevented them from proper vascular differentiation. In addition, MAPCs and BMCs used in this study failed to commit to cardiomyocytes in vitro (data not shown) or in vivo in the infarcted heart, given the lack of co-localization of the LacZ marker with cardiac $\alpha$-MHC (Figure 2K). Although some studies have suggested that, for instance, BM-derived progenitor cells may differentiate to immature cardiomyocytes (Orlic et al., 2001; Kajstura et al., 2005), many others have not shown such differentiation to occur (Wagers et al., 2002; Balsam et al., 2004; Murry et al., 2004) or demonstrated that this so-called differentiation is in fact due to fusion between grafted cells and host cardiomyocytes (Alvarez-Dolado et al., 2003; Reinecke et al., 2004).

Although $\mathrm{LacZ}^{+}$MAPCs did not directly contribute to vascular cells, they were consistently located in the immediate vicinity of BS-I lectin- and $\alpha$-SMA-positive vessels in the periphery of the infarct (Figure $2 \mathrm{~L}, \mathrm{M}$ ), putting them in a strategic position to affect vascular expansion. In line with such a hypothesis, at 8 weeks post-infarction, capillary density in the peri-infarct zone of hearts grafted with MAPCs was significantly $(p<0.05)$ higher than in the DMEM control, BMC and MEF groups (number of BS- $\mathrm{I}^{+}$capillaries $/ \mathrm{mm}^{2}: 1217 \pm 135,872 \pm 62,772 \pm 43$ and $827 \pm 176$ for MAPCs, DMEM, BMCs and MEFs, 
A
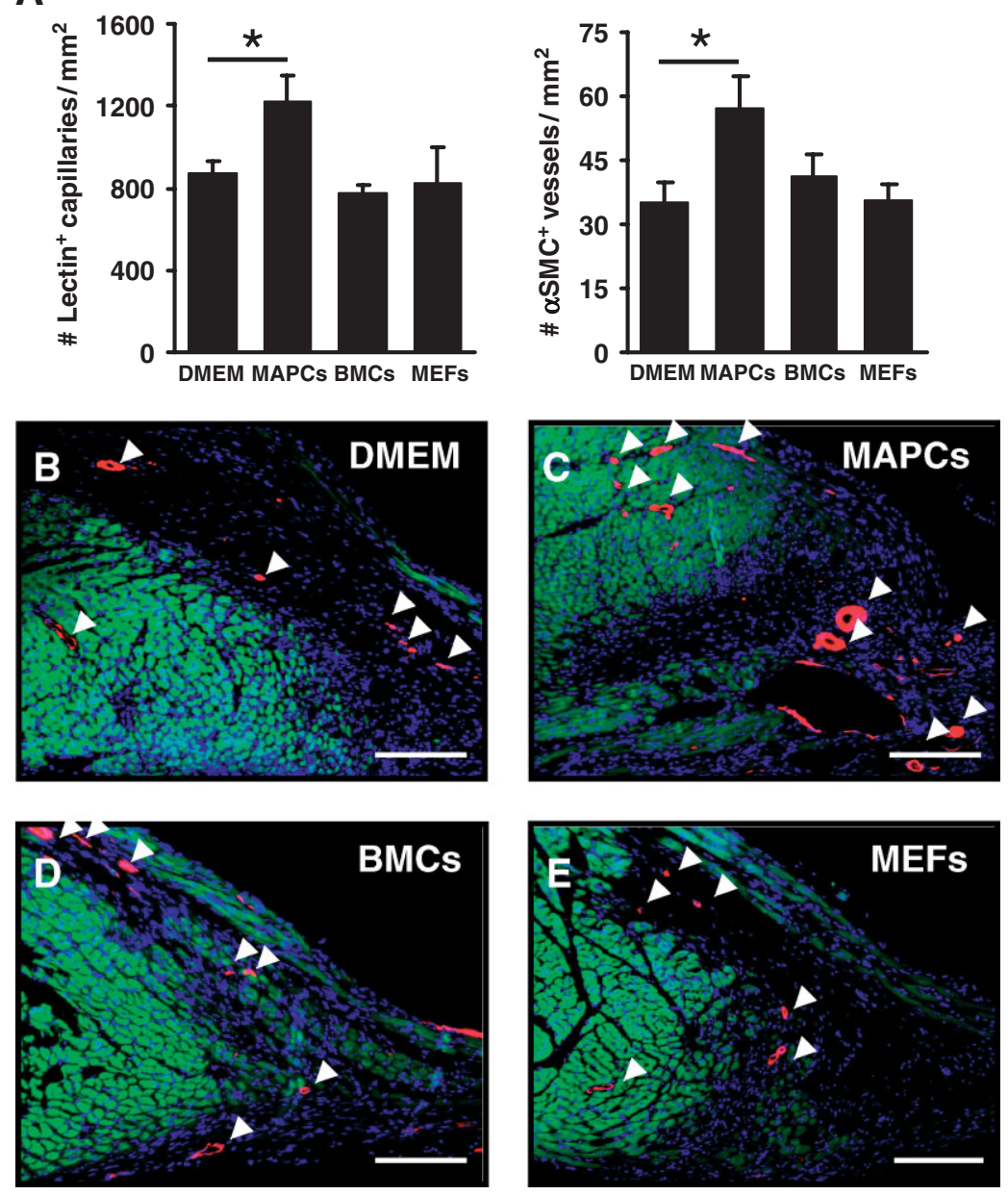

Figure 3. Significant capillary and arterial vessel expansion at 8 weeks in MAPC-treated hearts. (A) Counting results (mean \pm S.E.M) for capillaries $/ \mathrm{mm}^{2}$ and SMC-coated vessels $/ \mathrm{mm}^{2}$ in the peri-infarct area, 8 weeks following cell transplantation. A significant increase in the number of capillaries $/ \mathrm{mm}^{2}$ and SMC coated vessels $/ \mathrm{mm}^{2}$ was detected in the MAPC group only. For counting, capillaries were defined as small-sized vessels $\left(5-10 \mu \mathrm{m}\right.$ diameter). ${ }^{*} p<0.05$. Scale bars represent $50 \mu \mathrm{m}$. (B-E) $\alpha$-SMA immunostaining (red) of hearts from animals treated with DMEM (C), MAPCs (D), BMCs (E) and MEFs (F) 8 weeks following cell transplantation, here showing, in the peri-infarct zone and around the infarct border, a significant increase in the number of SMC coated vessels $/ \mathrm{mm}^{2}$ in the MAPC group. GFP-autofluorescent muscle demonstrates the viable myocyte containing area. Nuclear staining (blue), DAPI. * $p<0.05$. Scale bars represent $200 \mu \mathrm{m}$

Figure 4. MAPCs induce an inflammatory reaction. (A-C) Haematoxylin/eosin-stained sections of hearts from animals treated 1 week following transplantation of DMEM (A), BMCs (B), and MAPCs (C). Larger clusters of inflammatory cells were detected in the peri-infarct zone and around the infarct border (infarct border is indicated by black dotted lines) in the MAPC group than in the BMC group, whereas the DMEM group showed the lowest density of inflammatory cells. (D-G) Quantification of CD45-stained sections confirmed a significantly higher inflammatory infiltration in the MAPC (F) than in the BMC (E) or DMEM group (D). Results of the quantification are summarized in (G). Nuclear staining (blue), DAPI. Data in (G) represent mean \pm SEM. Scale bars represent $50 \mu \mathrm{m}(\mathrm{A}-\mathrm{C})$ or $25 \mu \mathrm{m}(\mathrm{D}-\mathrm{F})$

Figure 5. MAPC-conditioned medium induces in vitro EC and SMC proliferation and cardiomyocyte protection. (A) ELISAs on cell supernatants revealed a significant production of inflammatory (MCP-1) and angio/arteriogenic (VEGF, PDGF-BB, TGF $\beta_{1}$ ) factors by MAPCs, but not BMCs. Data represent mean \pm SEM and were calculated as [amount $(\mathrm{pg} / \mathrm{ml})$ in conditioned media - amount $(\mathrm{pg} / \mathrm{ml})$ in non-conditioned media]/100 000 cells (the non-conditioned medium corresponds to medium incubated in the absence of cells). The results summarize three independent experiments. ${ }^{*} p<0.05 ;{ }^{* *} p<0.01$; ${ }^{* * *} p<0.001$. (B, C) MAPC supernatant induced a significant increase in the proliferation rate of ECs and SMCs. ECs and SMCs were seeded at $375 \mathrm{cells} / \mathrm{cm}^{2}$ and 625 cells $/ \mathrm{cm}^{2}$, respectively, and cultured for $60 \mathrm{~h}$ in the presence of MAPC-conditioned medium (CM) or non-conditioned medium (NCM), at which time the cell number was enumerated in triplicate. The graphs (B) show the mean cell density $( \pm$ SEM) quantified in three independent experiments. ${ }^{*} p<0.05 ;{ }^{* *} p<0.01$. Representative pictures show the cell density differences after $60 \mathrm{~h}$ of culture in the presence of CM or NCM ( $\times 4$ magnification) (C). (D) Quantification of oligonucleosomal fragments by ELISA revealed a significant decrease in the apoptotic level of cardiomyocytes cultured for $24 \mathrm{~h}$ under hypoxic and serum-deprived culture conditions in MAPC-conditioned medium (CM). The graph shows the percentage of apoptosis decrease $( \pm$ SEM) in cardiomyocytes cultured in $\mathrm{CM}$ vs. non-conditioned medium (NCM). The results summarize three independent experiments in which cells were seeded and counted in quadruplicate. ${ }^{*} p<0.05 ;{ }^{* *} p<0.01$ 


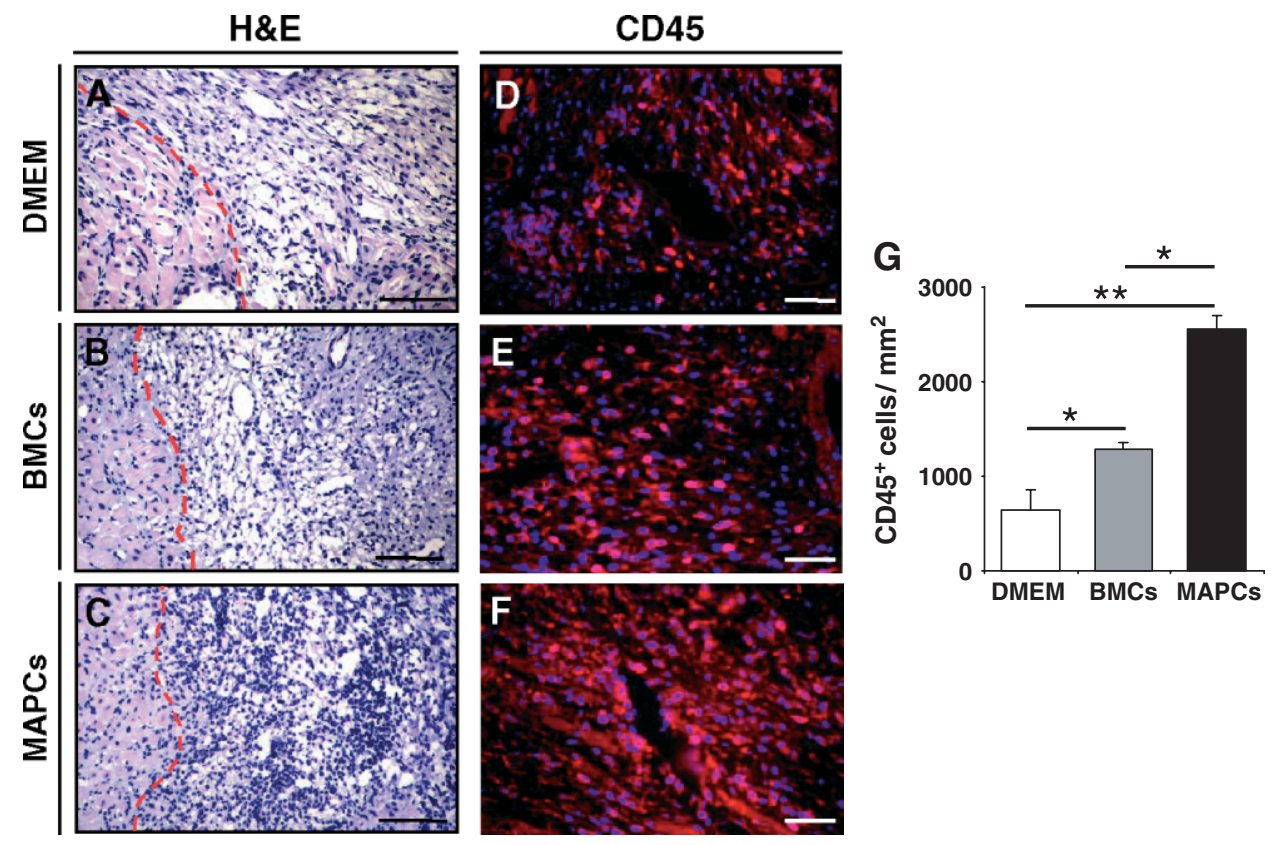

Figure 4.
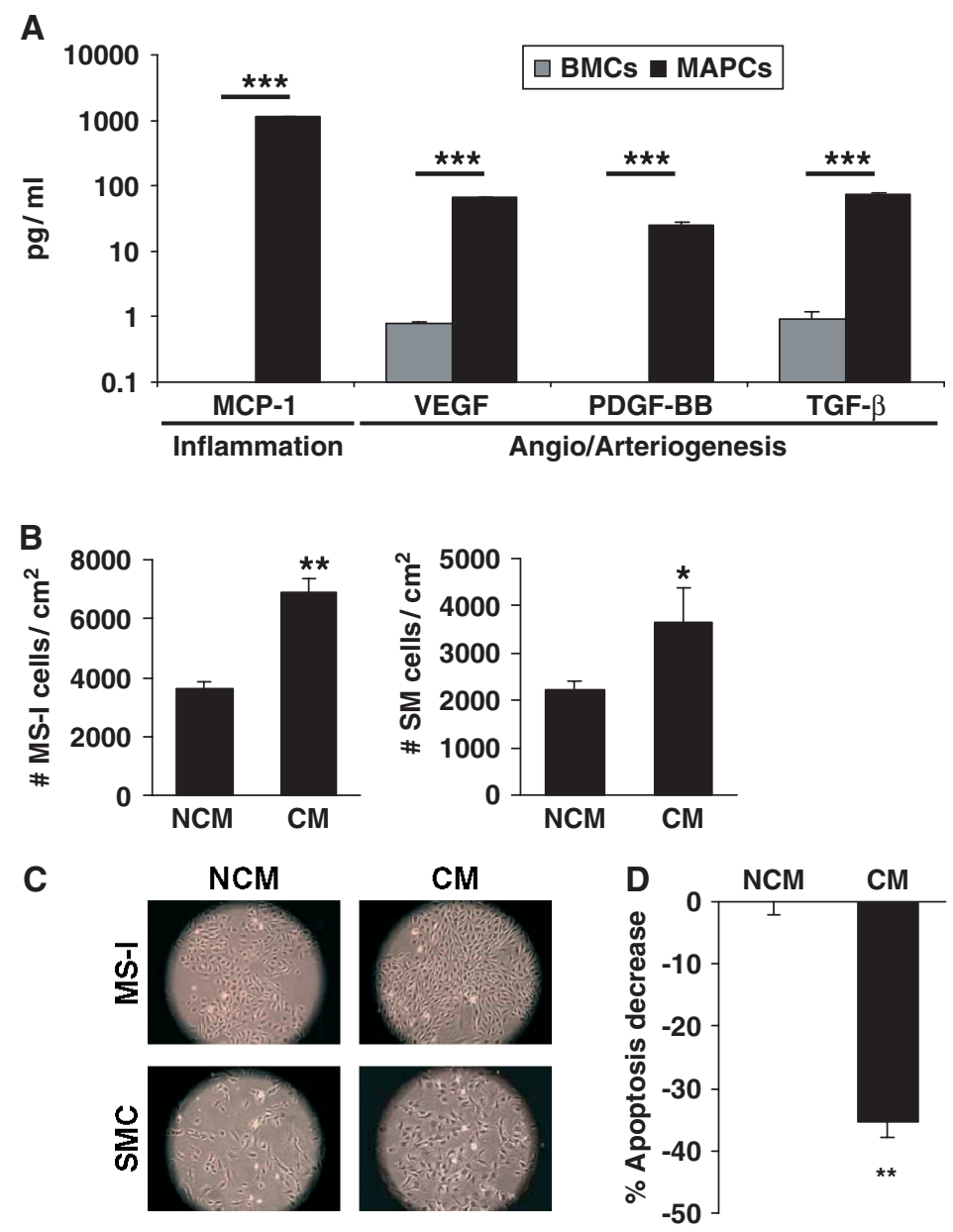

Figure 5. 
respectively; Figure 3A). Furthermore, unlike the other cell types, MAPCs significantly $(p<0.05)$ stimulated the growth of arteries and arterioles in the viable peri-infarct zone and around the infarct border, thereby likely expanding the capacity of larger conductance vessels (number of $\alpha$-SMA ${ }^{+}$vessels $/ \mathrm{mm}^{2}: 57 \pm 7,35 \pm 5,41 \pm 5$ and $36 \pm 4$ for MAPCs, DMEM, BMCs and MEFs, respectively; Figure $3 \mathrm{~B}-\mathrm{E})$. As inflammation is known to contribute to neovascularization and infarct healing, the degree of inflammation was analysed at 7 days following LAD ligation, when the inflammatory process is at its peak levels (Heymans et al., 1999). There was a moderate inflammatory response in the periphery and around the border of the infarct in BMC-grafted hearts compared to DMEM, but a significantly higher increase in the number of inflammatory cells in MAPC-transplanted hearts, as compared to BMCs and DMEM (number of CD $45^{+}$cells $/ \mathrm{mm}^{2}$ : $2562 \pm 138,1290 \pm 62,643 \pm 220$ for MAPCs, BMCs and DMEM, respectively; $p<0.05$ vs. DMEM; $p<0.05$ for MAPCs vs. BMCs; Figure 4).

As the short-term presence of MAPCs resulted in significant reduction in infarct size and a robust vascular response, while they did not differentiate into vascular cells or cardiomyocytes in vivo, we hypothesized that they could have a trophic effect on neovascularization and cardiac protection, as previously shown with other cell types (Mangi et al., 2003; Galli et al., 2005; Gnecchi et al., 2005). Moreover, BMCs did not exert any effect on vascular expansion and had a less pronounced effect on infarct size and cardiac function, potentially due to a less favourable cytokine secretion profile. Therefore, we compared the cytokine profile of both cell populations in vitro. Compared with BMCs, MAPCs produced significantly higher levels of the endothelial growth factor VEGF, as well as PDGF-BB and TGF $\beta_{1}$, known to be involved in SMC proliferation/differentiation. In addition, high levels of macrophage chemoattractant protein (MCP)-1, a potent inflammatory stimulator, known to recruit monocytes which are needed for the initiation of angiogenesis (Kinnaird et al., 2004), were secreted by MAPCs but not by BMCs (Figure 5A). Interestingly, secretion of bFGF, shown by others to impact angiogensis in the infarcted heart (Kamihata et al., 2001; Galli et al., 2005), was equivalent in BMC and MAPC supernatants (data not shown). Moreover, MAPC-conditioned media could induce a significant increase in the proliferation rate of murine endothelial and smooth muscle cells ( $90 \pm 3 \%$ increase for ECs $(p<0.01)$ and a $69 \pm 12 \%$ increase for SMCs $(p<0.05)$ (Figure 5B,C). While the beneficial effects of the cells on neovascularization may have protected the viable myocardium at risk, some of the protection could also be due to a direct survival effect on cardiomyocytes, a mechanism previously shown by others to be important for limiting scar size (Gnecchi et al., 2005). In order to evaluate a possible cardioprotective effect of the implanted cells, we compared the degree of apoptosis induced by hypoxia $\left(0.5 \% \mathrm{O}_{2}\right)$ and serum deprivation between cultures of cardiomyocytes incubated with
MAPC-conditioned medium (CM) and control media (NCM). When cultured in the presence of MAPC CM, a significant decrease of $35 \pm 3 \%(p<0.01)$ in the fraction of apoptotic cardiomyocytes was seen compared to NCM (Figure 5D).

In conclusion, we demonstrate that MAPCs are superior to BMCs to decrease infarct size and improve LV function following AMI in mice. These favourable effects were likely, or at least partially, due to a trophic effect on neovascularization and cardioprotection of the area at risk. Therefore, it will be interesting to determine whether this superior effect is also apparent in larger animal models and, ultimately, in patients with AMI.

\section{Acknowledgements}

This work was funded by grants from the National Institutes of Health (R01 HL67828 to J.Z., R01 HL69119 to C.M.V.), the American Heart Association (0525748Z to B.P., 0520133Z to A.L.), the Belgian American Educational Foundation (to A.L.) and Athersys Inc. (to C.M.V.). We thank X. L. Aranguren (University of Navarra, Spain) for technical advice for staining. The HL-1 cardiomyocyte cell-line was kindly provided by $\mathrm{Dr}$ Claycomb (Louisiana State University Medical Center, USA).

\section{References}

Alvarez-Dolado M, Pardal R, Garcia-Verdugo JM, et al. 2003; Fusion of bone-marrow-derived cells with Purkinje neurons, cardiomyocytes and hepatocytes. Nature 425(6961): 968-973.

Andersson G, Denaro M, Johnson K, et al. 2003; Engraftment of retroviral EGFP-transduced bone marrow in mice prevents rejection of EGFP-transgenic skin grafts. Mol Ther 8(3): 385-391.

Balsam LB, Wagers AJ, Christensen JL, et al. 2004; Haematopoietic stem cells adopt mature haematopoietic fates in ischaemic myocardium. Nature 428(6983): 668-673.

Brazelton TR, Blau HM. 2005; Optimizing techniques for tracking transplanted stem cells in vivo. Stem Cells 23(9): 1251-1265.

Chockalingam A, Balaguer-Vintro I, Achutti A, et al. 2000; The World Heart Federation's white book: impending global pandemic of cardiovascular diseases: challenges and opportunities for the prevention and control of cardiovascular diseases in developing countries and economies in transition. Can J Cardiol 16(2): 227-229.

Davani S, Marandin A, Mersin N, et al. 2003; Mesenchymal progenitor cells differentiate into an endothelial phenotype, enhance vascular density, and improve heart function in a rat cellular cardiomyoplasty model. Circulation 108(suppl 1): II253-258.

Francis GS, Tang WH. 2003; Pathophysiology of congestive heart failure. Rev Cardiovasc Med 4(suppl 2): S14-20.

Galli D, Innocenzi A, Staszewsky L, et al. 2005; Mesoangioblasts, vessel-associated multipotent stem cells, repair the infarcted heart by multiple cellular mechanisms: a comparison with bone marrow progenitors, fibroblasts, and endothelial cells. Arterioscler Thromb Vasc Biol 25(4): 692-697.

Gnecchi M, He H, Liang OD, et al. 2005; Paracrine action accounts for marked protection of ischaemic heart by Akt-modified mesenchymal stem cells. Nat Med 11(4): 367-368.

Heymans S, Luttun A, Noyens D, et al. 1999; Inhibition of plasminogen activators or matrix metalloproteinases prevents cardiac rupture but impairs therapeutic angiogenesis and causes cardiac failure. Nat Med 5(10): 1135-1142.

Hogan B. 1994; Manipulating the Mouse Embryo: A Laboratory Manual. Cold Spring Harbor Laboratory Press: Cold Spring Harbor, NY.

Hong KM, Najjar H, Hawley M, et al. 2004; Quantitative realtime PCR with automated sample preparation for diagnosis 
and monitoring of cytomegalovirus infection in bone marrow transplant patients. Clin Chem 50(5): 846-856.

Jackson KA, Majka SM, Wang H, et al. 2001; Regeneration of ischaemic cardiac muscle and vascular endothelium by adult stem cells. J Clin Invest 107(11): 1395-1402.

Jiang Y, Jahagirdar BN, Reinhardt RL, et al. 2002; Pluripotency of mesenchymal stem cells derived from adult marrow. Nature 418(6893): 41-49.

Kajstura J, Rota M, Whang B, et al. 2005; Bone marrow cells differentiate in cardiac cell lineages after infarction independently of cell fusion. Circ Res 96(1): 127-137.

Kamihata H, Matsubara H, Nshive T, et al. 2001; Implantation of bone marrow mononuclear cells into ischaemic myocardium enhances collateral perfusion and regional function via side supply of angioblasts, angiogenic ligands, and cytokines. Circulation 104(9): 1046-1052.

Kinnaird T, Stabile E, Burnett MS, et al. 2004; Local delivery of marrow-derived stromal cells augments collateral perfusion through paracrine mechanisms. Circulation 109(12): 1543-1549.

Kisseberth WC, Brettingen NT, Lonsa JK, et al. 1999; Ubiquitous expression of marker transgenes in mice and rats Dev Biol 214(1): $128-138$.

Kocher AA, Schuster MD, Seaboles MJ, et al. 2001; Neovascularization of ischaemic myocardium by human bone-marrow-derived angioblasts prevents cardiomyocyte apoptosis, reduces remodelling and improves cardiac function. Nat Med 7(4): 430-436.

Mangi AA, Noiseux N, Kong D, et al. 2003; Mesenchymal stem cells modified with Akt prevent remodelling and restore performance of infarcted hearts. Nat Med 9(9): 1195-1201.

Menasche P. 2005; Skeletal myoblast for cell therapy. Coron Artery Dis 16(2): 105-110.
Murry CE, Field LJ, Menasche P, et al. 2005; Cell-based cardiac repair: reflections at the 10-year point. Circulation 112(20): 3174-3183.

Murry CE, Soonpaa MH, Reinecue H, et al. 2004; Haematopoietic stem cells do not transdifferentiate into cardiac myocytes in myocardial infarcts. Nature 428(6983): 664-668.

Nakamura Y, Yoshiyama M, Omura T, et al. 2002; Transmitral inflow pattern assessed by Doppler echocardiography in angiotensin II type 1 A receptor knockout mice with myocardial infarction. Circ $J$ 66(2): 192-196.

Orlic D, Kajstura J, Chimenb S, et al. 2001; Bone marrow cells regenerate infarcted myocardium. Nature 410(6829): 701-705.

Reinecke H, Minami E, Pappa V, et al. 2004; Evidence for fusion between cardiac and skeletal muscle cells. Circ Res 94(6): e56-60.

Reyes M, Dudek A, Jahagirdar B, et al. 2002; Origin of endothelial progenitors in human postnatal bone marrow. $J$ Clin Invest 109(3): 337-346.

Shimizu K, Sugiyama S, Abawa M, et al. 2001; Host bone-marrow cells are a source of donor intimal smooth muscle-like cells in murine aortic transplant arteriopathy. Nat Med 7(6): 738-741.

Tolar J, J O'Shaughnessy M, Panasnaltsis Mortan A, et al. 2006; Host factors that impact the biodistribution and persistence of multipotent adult progenitor cells. Blood 107(10): 4182-4188.

Tomita S, Li RK, Weisel RD, et al. 1999; Autologous transplantation of bone marrow cells improves damaged heart function. Circulation 100(19 suppl): II247-256.

Urbich C, Dimmeler S. 2004; Endothelial progenitor cells functional characterization. Trends Cardiovasc Med 14(8): 318-322.

Wagers AJ, Sherwood RI, Christensen JL, et al. 2002; Little evidence for developmental plasticity of adult haematopoietic stem cells. Science 297(5590): 2256-2259. 